\title{
Identification of a Novel a-herpesvirus Associated with Ulcerative Stomatitis in Donkeys
}

\author{
Vito Martella, Gianvito Lanave, Michele Camero, Vittorio Larocca, Eleonora Lorusso, \\ Cristiana Catella, Paolo Capozza, Maria Tempesta, Canio Buonavoglia
}

An outbreak of ulcerative stomatitis with was observed in a donkey (Equus asinus) dairy herd. Similar lesions were also observed on the dams' udders and, sporadically, in genital areas. The lesions typically resolved in 1-3 weeks. An a-herpesvirus, Varicellovirus, genetically related to equid herpesvirus type 3 , was identified.

$\mathrm{V}$ esicular stomatitis is a consequential disease of equids. Vesicular stomatitis virus (family Rhabdoviridae, genus Vesiculovirus) is a major infectious agent with zoonotic potential that is common in the Americas. A few other infectious viral agents (equine arteritis virus, bunyavirus, caliciviruses, equine adenoviruses, and herpesviruses) have been associated with this condition in horses, but on several occasions the etiology of vesicular and ulcerative stomatitis remains undiagnosed (1). Noninfectious etiology may include plantand drug-related toxicoses and photosensitization (1).

In October 2011, an outbreak of ulcerative stomatitis started in a donkey (Equus asinus) dairy herd, comprising 106 animals, located in the prefecture of Bari, Apulia region, Italy. The outbreak appeared related to the introduction of a pregnant female 8 weeks before the onset of the disease. The mare was clinically healthy and in good physical condition at arrival and gave birth to a foal after 7 weeks. Clinical signs developed in neither the dam nor the foal.

Initially, the outbreak affected 34 animals (17 lactating mares with their foals). This group was separated with wood fences from the other animals, but not strictly. Fever and small nodular lesions, evolving into painful ulcers, were observed on the oral mucosa, tongue, and skin around the lips of young animals (2 weeks-4 months old) (Figure 1). Similar lesions were also observed sporadically on the dams' udders and genital

Author affiliation: University of Bari, Valenzano, Italy

DOI: https://doi.org/10.3201/eid2612.200201 areas. The lesions typically resolved in 1-3 weeks. The herd owner reported weight loss in foals and interruption of lactation in dams. Two weeks after onset in the original group, the disease was observed in a separate group of animals, comprising 63 adult or yearling females and 5 yearling males. In this group, however, oral lesions were observed only in 5 yearlings and 1 mare. A third group of 4 adult males was kept apart from the other animals and was not affected by the disease.

Oral swab and serum samples collected from 8 animals with acute disease were sent to the laboratories at the University of Bari (Valenzano, Italy) for virologic investigation. Using an electron microscope, we observed herpesvirus-like particles in the oral swabs and detected herpesvirus DNA using consensus herpesvirus primers for the DNA polymerase and inverse terminase $(2,3)$. We used BLASTn (https:// blast.ncbi.nlm.nih.gov) to search the GenBank genetic sequence database and found the virus to be highly related to equid herpesvirus (EHV) 3 in the DNA polymerase $(93.35 \% \mathrm{nt}$ identity) and terminase (90.71\% nt identity) regions.

We isolated the virus onto equine dermal cells from oral swab specimens. The virus was titrated and used for screening serum samples collected from the donkeys in virus neutralization assays. We detected specific neutralizing antibodies in the serum samples collected from approximately three quarters (80/106) of the animals 2 months after the beginning of the outbreak but not in the serum samples of 8 animals with acute infection, suggesting seroconversion.

To sequence the DNA of the isolate, we performed next generation sequencing using the Illumina MiSeq platform (https://www.illumina.com) and used Nextera XT (Illumina) for library preparation. We obtained the full genome sequence $(147,607$ bp) of asinine herpesvirus (AsHV) strain AsHV/ Bari/2011/740 and annotated it using the software ORF Finder (https:/ / www.bioinformatics.org). 

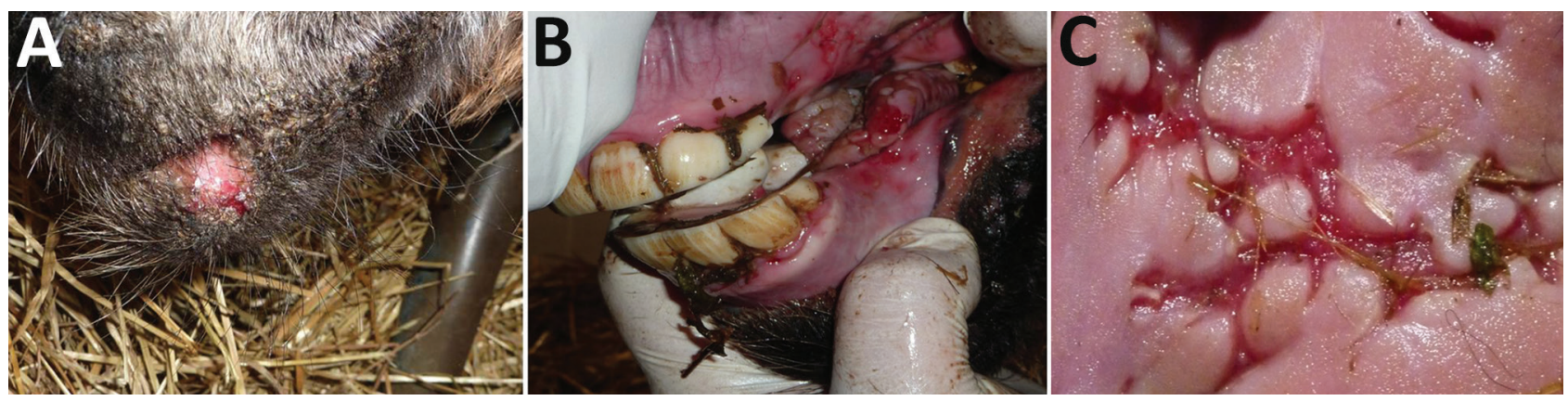

Figure 1. Foals with herpesviral infection, part of an outbreak of ulcerative stomatitis in a donkey dairy herd, Bari, Italy. Ulcerative lesions were observed on the lips (A), oral mucosa (B), and tongue (C).

On full genome sequence analysis, strain AsHV/ Bari/2011/740 appeared genetically related $(87.02 \%$ nt identity) to EHV-3 strain AR/2007/C3A (accession no. KM051845) (subfamily a-Herpesviridae, genus Varicellovirus). Three different full-length gene targets (glycoproteins B, C, and D) of strain AsHV/ Bari/2011/740 were aligned with cognate sequences representative of the genus Varicellovirus listed by the International Committee on Taxonomy of Viruses (https://talk.ictvonline.org) by using Geneious software version 9.1.8 (Biomatters Ltd., https://www. geneious.com) and the MAFFT algorithm (4). We performed phylogenetic analyses with MEGAX software (https://www.megasoftware.net) (5) using the

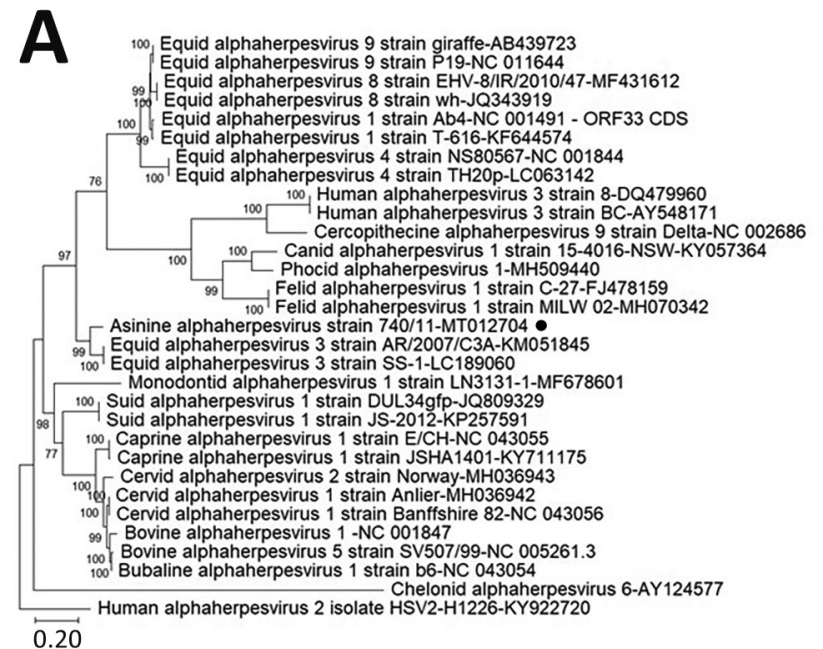

0.20

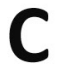

60. Equid alphaherpesvirus 1 strain Ab4-NC 001491 60. Equid alphanerpesvirus 1 strain Ab4-NC 001491 Equid alphaherpesvirus 9 strain giraffe-AB453826

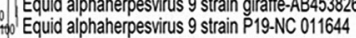
Equid alphaherpesvirus 8 strain EHV-8/R/2010/47-MF431612 100 Equid alphaherpesvirus 8 strain wh- -0343919

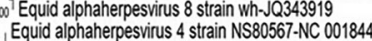
${ }_{100}$ Equid alphaherpesvirus 4 strain TH20p-LC063142 Cercopithecine alphaherpesvirus 9 strain Delta-NC 002686

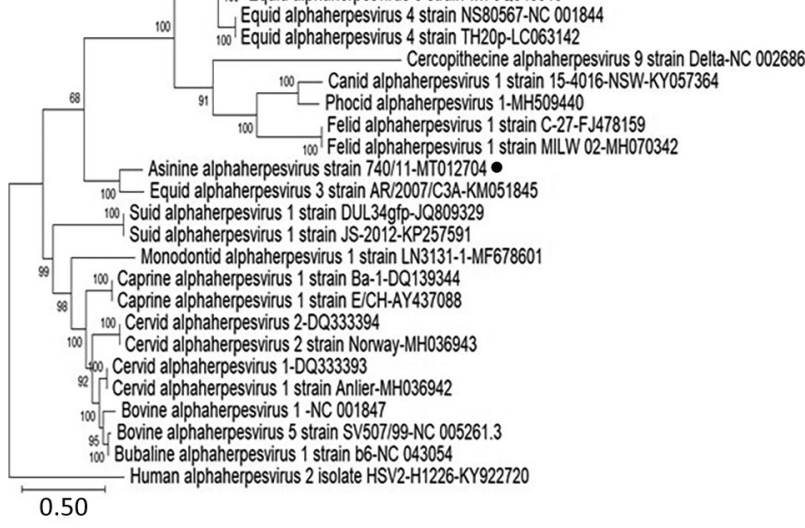

B

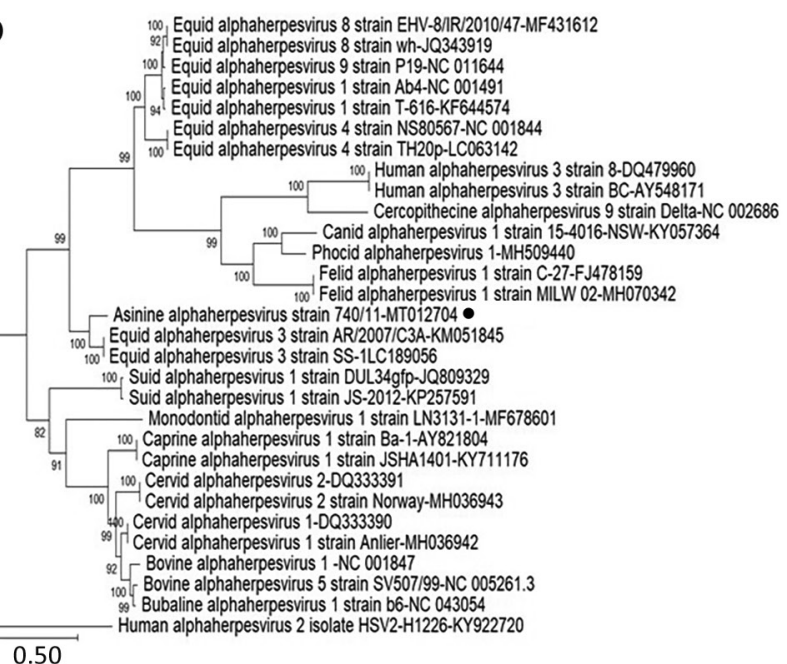

Figure 2. Phylogenetic trees based on the full-length nucleotide sequence of different target genes from the AsHV/ Bari/2011/740 strain isolated from a donkey dairy herd, Bari, Italy (black dots), or retrieved from the International Committee on Taxonomy of Viruses database. A) Glycoprotein B; B) glycoprotein C; C) glycoprotein D. Posterior output of the tree was derived from maximum-likelihood inference using a general time-reversible model, a proportion of invariable sites, a gamma distribution of rate variation across sites, and a subsampling frequency of 1,000. Posterior probability values $>70 \%$ are indicated on the tree nodes. Human alphaherpesvirus 2 isolate HSV-2-H1226 (genus Simplesvirus) strain (GenBank accession no. KY922720) was used as an outgroup. Scale bars indicate nucleotide substitutions per site. AsHV, asinine herpesvirus. 
Table. List of equid herpesviruses recognized in the ICTV database or other sources*

\begin{tabular}{lcccc}
\hline Species & Subfamily & Related to & Other designations & Source \\
\hline EHV-1 & Alpha & NA & Equine abortion & ICTV \\
EHV-2 & Gamma & NA & NA & Coital exantema \\
EHV-3 & Alpha & NA & Equine rhinopneumonitis & ICTV \\
EHV-4 & Alpha & NA & NA & ICTV \\
EHV-5 & Gamma & NA & AsHV-1 & ICTV \\
EHV-6 & Alpha & EHV-3 & AsHV-2 & NA \\
EHV-7 & Gamma & EHV-2/EHV-5 this study \\
EHV-8 & Alpha & EHV-1 & NA & ICTV \\
AsHV-4 & Gamma & EHV-2/EHV-5 & ICTV \\
AsHV-5 & Gamma & EHV-2/EHV-5 & $(11)$ \\
AsHV-6 & Gamma & EHV-2/EHV-5 & Z & $(11)$ \\
EHV-9 & Alpha & EHV-1 & Zebra, gazelle, giraffe herpesviruses & ICTV \\
${ }^{*}$ AsHV, asinine herpesvirus; EHV, equine herpesvirus; ICTV, International Committee on Taxonomy of Viruses; NA, not applicable.
\end{tabular}

maximum-likelihood method with the general timereversible model, a proportion of invariant sites, and a discrete gamma distribution (5 categories) to model evolutionary rate differences among sites, and bootstrap analyses with 1,000 pseudoreplicate datasets. In the consensus phylogenetic trees (Figure 2), strain AsHV/Bari/2011/740 was closely related to EHV-3 sequences and distantly related to other members of the genus Varicellovirus. We deposited the full-genome sequence of strain AsHV/Bari/2011/740 in the GenBank database (accession no. MT012704).

To date, several herpesviruses have been discovered in donkeys (Table). AsHV type 1, also called EHV6 , is an a-herpesvirus associated with ulcerative lesions (6). AsHV-2 (EHV-7) is a $\gamma$-herpesvirus identified in leukocytes of healthy animals (7). AsHV-3 (EHV-8) was isolated from the nose of immunodepressed animals (8) and was classified as an a-herpesvirus on the basis of the glycoprotein $\mathrm{G}$ sequence and poor antigenic cross-reactivity with EHV-1 and EHV-4 (8-10). AsHV-4, -5 , and -6 are $\gamma$-herpesviruses identified from donkeys with interstitial pneumonitis $(2,11)$.

We report the detection and isolation of a novel AsHV from an outbreak of vesicular and ulcerative stomatitis and mammillitis in a donkey dairy herd. By comparing it with other herpesvirus sequences from the databases, we identified 3 targets (glycoproteins B, C, and D) for which the sequences were available across all the varicelloviruses listed in the ICTV database and that were used for phylogenetic analysis. In these analyses, the AsHV strain appeared similar to EHV-3. By reviewing the literature, we found another donkey herpesvirus, AsHV-1, genetically related to EHV-3 on the basis of restriction enzyme and hybridization analyses (6). AsHV-1 was originally isolated from the vesicular and erosive lesions of the muzzle of a foal and the external genitalia and udder of its dam (12). Unfortunately, the prototype AsHV-1 is no longer available and it is not possible to determine its genome sequence for precise comparison (G.F.
Browning, pers. comm.). It is possible that AsHV/ Bari/2011 is actually an AsHV-1 strain, although this possibility cannot be confirmed.

Overall, based on the chronology of the health events observed in the herd, the tendency of herpesviruses to reactivate from latent infection under stress conditions, and the seroconversion observed in the monitored animals, we hypothesized that the newly introduced mare was the vehicle for herpesvirus infection in the herd, although this possibility could not be conclusively confirmed. Also, we screened only 8 animals during the acute phase of the disease, and we do not have an exact picture of the immunological status of the animals before the onset of the disease.

In conclusion, we identified a novel AsHV, genetically related to EHV-3, from an outbreak of infectious ulcerative stomatitis in donkey foals. These findings extend the spectrum of pathologies potentially attributable to herpesviruses in donkeys. Considering the nature and shape of the lesions, the virus should be included in the differential diagnosis of vesicular and ulcerative stomatitis among equids. Also, it needs to be determined whether the novel AsHV can be transmitted to horses.

\section{Acknowledgments}

We are grateful to Aristide Maggiolino for collecting the animal samples and to Carlo Armenise and Arturo Gentile for their support in laboratory work.

The authors dedicate this work to the memory of Professor Leland Eugene "Skip" Carmichael (June 15 1930-July 27 2020), College of Veterinary Medicine, Cornell University, Ithaca, NY, USA, for his contribution to the growth of veterinary virology, his decades-long friendship and scientific collaboration, and his profound sense of humanity.

\section{About The Author}

Dr. Martella is a full professor in the Department of Veterinary Medicine, University of Bari, Italy. His research 
is focused on diagnosis, epidemiology, and characterization of human and animal viruses, with particular interest for zoonotic viruses.

\section{References}

1. McCluskey BJ, Mumford EL. Vesicular stomatitis and other vesicular, erosive, and ulcerative diseases of horses. Vet Clin North Am Equine Pract. 2000;16:457-69, viii-ix. https:/ / doi.org/10.1016/s0749-0739(17)30089-5

2. Kleiboeker SB, Chapman RK. Detection of equine herpesvirus 3 in equine skin lesions by polymerase chain reaction. J Vet Diagn Invest. 2004;16:74-9. https:/ / doi.org/ $10.1177 / 104063870401600113$

3. VanDevanter DR, Warrener P, Bennett L, Schultz ER, Coulter S, Garber RL, et al. Detection and analysis of diverse herpesviral species by consensus primer PCR. J Clin Microbiol. 1996;34:1666-71. https:/ / doi.org/10.1128/ JCM.34.7.1666-1671.1996

4. Katoh K, Misawa K, Kuma K, Miyata T. MAFFT: a novel method for rapid multiple sequence alignment based on fast Fourier transform. Nucleic Acids Res. 2002;30:3059-66. 10.1093/nar/gkf436 https:/ / doi.org/10.1093/nar/gkf436

5. Kumar S, Stecher G, Li M, Knyaz C, Tamura K. MEGA X: molecular evolutionary genetics analysis across computing platforms. Mol Biol Evol. 2018;35:1547-9. https://doi.org/ 10.1093/molbev/msy096

6. Browning GF, Ficorilli N, Studdert MJ. Asinine herpesvirus genomes: comparison with those of the equine herpesviruses. Arch Virol. 1988;101:183-90. https://doi.org/ 10.1007/BF01310999

7. Bell SA, Pusterla N, Balasuriya UB, Mapes SM, Nyberg NL, MacLachlan NJ. Isolation of a gammaherpesvirus similar to asinine herpesvirus-2 (AHV-2) from a mule and a survey of mules and donkeys for AHV-2 infection by real-time PCR. Vet Microbiol. 2008;130:176-83. https://doi.org/10.1016/ j.vetmic.2007.12.013

8. Crabb BS, Allen GP, Studdert MJ. Characterization of the major glycoproteins of equine herpesviruses 4 and 1 and asinine herpesvirus 3 using monoclonal antibodies. J Gen Virol. 1991;72:2075-82. https:/ / doi.org/10.1099/ 0022-1317-72-9-2075

9. Ficorilli N, Studdert MJ, Crabb BS. The nucleotide sequence of asinine herpesvirus 3 glycoprotein $\mathrm{G}$ indicates that the donkey virus is closely related to equine herpesvirus 1. Arch Virol. 1995;140:1653-62. https:/ / doi.org/10.1007/ BF01322539

10. Paweska JT, Gerdes T, Van Heerden J. Serological relationship between a donkey alphaherpesvirus (isolate M7/91) and equid herpesvirus type 1 and 4. J S Afr Vet Assoc. 1994;65:64-6.

11. Kleiboeker SB, Schommer SK, Johnson PJ, Ehlers B, Turnquist SE, Boucher M, et al. Association of two newly recognized herpesviruses with interstitial pneumonia in donkeys (Equus asinus). J Vet Diagn Invest. 2002;14:273-80. PubMed https://doi.org/10.1177/104063870201400401

12. Burrows R. Discussion. In: Bryans JT, Gerber H, editors. Equine infectious diseases III. Proceedings of the Third International Conference on Equine Infectious Diseases; 1972 Jul 17-21; Paris. Basel: S Karger; 1973

Address for correspondence: Vito Martella, Department of Veterinary Medicine, University of Bari, S.p. per Casamassima km 3, 70010, Valenzano, Bari, Italy; email: vito.martella@uniba.it

\section{EID Podcast: WWI and the 1918 Flu Pandemic \\ CDC's Dr. Terence Chorba \\ discusses his EID cover art essay about the 1918 flu \\ pandemic and the WWI painting by John Singer Sargent.}

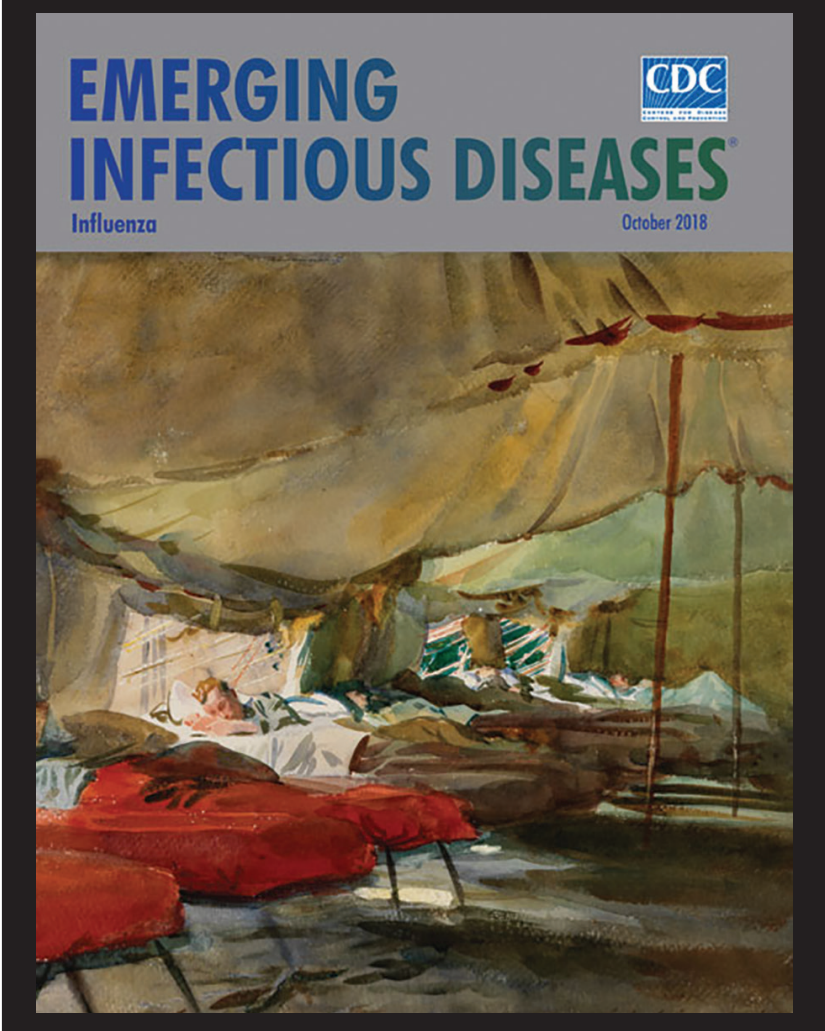

Visit our website to listen: https://tools.cdc.gov/medialibrary/ index.aspx\#/media/id/393699

EMERGING INFECTIOUS DISEASES 